\title{
The Spanish version of the Fatigue Assessment Scale: Reliability and validity assessment in postpartum women
}

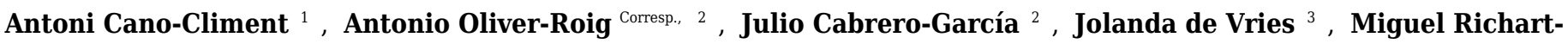 \\ Martínez $^{2}$ \\ ${ }^{1}$ Hospital General d'Ontinyent, Conselleria de Sanitat Universal i Salut Pública, Ontinyent, Valencia, Spain \\ 2 Department of Nursing, University of Alicante, San Vicent del Raspeig, Alicante, Spain \\ 3 CoRPS Center of Research on Psychology in Somatic Diseases, Department of Medical and Clinical Psychology, University of Tilburg, Tilburg, Netherlands \\ Corresponding Author: Antonio Oliver-Roig \\ Email address: Antonio.Oliver@ua.es
}

Background: Fatigue is the most widely reported symptom by women during pregnancy, labour, the postpartum period, and early parenting. The objective was to translate the Fatigue Assessment Scale (FAS) into Spanish and assess its psychometric properties.

Methods: Instrumental Design. The FAS was translated into Spanish (FAS-e) using forward and back translation. A convenience sample was constituted with 870 postpartum women recruited at discharge from 17 public hospitals in Eastern Spain. Data was obtained from clinical records and self-administered questionnaires at discharge. Internal consistency, factor structure, comparisons between known groups and correlations with other variables were assessed.

Results: Cronbach's alpha coefficient was .80. Findings on the dimensionality of the FAS-e scale indicated that it was sufficiently unidimensional. FAS-e scores were higher among women who had undergone caesarean births $(p<.05)$, had a higher level of postpartum pain $(p<.01)$, experienced difficulties during breastfeeding $(p<.01)$ and had lower levels of self-efficacy for breastfeeding $(p<.01)$.

Conclusions: An equivalent Spanish version of the FAS was obtained with good reliability and validity properties. FAS-e is an appropriate tool to measure postpartum fatigue. 


\section{The Spanish version of the Fatigue Assessment Scale:}

2 Reliability and Validity assessment in postpartum

3 women.

4 Antoni Cano-Climent ${ }^{1}$ Antonio Oliver-Roig ${ }^{2}$, Julio Cabrero-García ${ }^{2}$, Jolanda deViers ${ }^{3}$, Miguel

5 Richart-Martínez².

$6{ }^{1}$ Hospital General d'Ontinyent, Conselleria de Sanitat Universal i Salut Pública, Ontinyent,

7 Valencia, Spain.

$8{ }^{2}$ Department of Nursing, University of Alicante, San Vicent del Raspeig, Alicante, Spain.

$9{ }^{3}$ CoRPS Center of Research on Psychology in Somatic Diseases, Department of Medical and 10 Clinical Psychology, University of Tilburg, Tilburg, Netherlands.

11

12 Corresponding Author:

13 Antonio Oliver-Roig ${ }^{2}$

14 Email address: $\underline{\text { Antonio.Oliver@ua.es }}$ 


\section{$16 \underline{\text { Abstract }}$}

17 Background: Fatigue is the most widely reported symptom by women during pregnancy, labour, 18 the postpartum period, and early parenting. The objective was to translate the Fatigue Assessment 19 Scale (FAS) into Spanish and assess its psychometric properties.

20 Methods: Instrumental Design. The FAS was translated into Spanish (FAS-e) using forward and 21 back translation. A convenience sample was constituted with 870 postpartum women recruited at 22 discharge from 17 public hospitals in Eastern Spain. Data was obtained from clinical records and 23 self-administered questionnaires at discharge. Internal consistency, factor structure, comparisons 24 between known groups and correlations with other variables were assessed.

25 Results: Cronbach's alpha coefficient was .80. Findings on the dimensionality of the FAS-e scale 26 indicated that it was sufficiently unidimensional. FAS-e scores were higher among women who 27 had undergone caesarean births $(\mathrm{p}<.05)$, had a higher level of postpartum pain $(\mathrm{p}<.01)$, 28 experienced difficulties during breastfeeding $(\mathrm{p}<.01)$ and had lower levels of self-efficacy for 29 breastfeeding $(\mathrm{p}<.01)$.

30 Conclusions: An equivalent Spanish version of the FAS was obtained with good reliability and 31 validity properties. FAS-e is an appropriate tool to measure postpartum fatigue. 


\section{Introduction.}

34 Fatigue is a complex, multi-causal, multi-dimensional, non-specific, and subjective phenomenon

35 that has no widely accepted single definition (Tiesinga, Dassen, \& Halfens, 1998). Fatigue appears

36 when demands of a process or situation exceed available resources and recovery mechanisms are

37 not sufficient (Aaronson et al., 1999). One attempt to standardize the definition of fatigue can be

38 found in the Medical Subject Headings (MeSH) controlled vocabulary, where fatigue is defined as

39 "the state of weariness following a period of exertion, mental or physical, characterized by a

40 decreased capacity for work and reduced efficiency to respond to stimuli" (National Library of

41 Medicine, 2016).

42 Fatigue during pregnancy, labour, the postpartum period, and early parenthood is a common 43 experience. Its prevalence is highly variable, ranging between $15 \%$ and $76 \%$ (Cheng \& Li, 2008), 44 depending on the measurement instrument, culture, and period measured (Cheng et al., 2014).

45 Fatigue is an important symptom that can interfere in the transition to motherhood (McQueen \& 46 Mander, 2003). During pregnancy, fatigue is associated with depression, anxiety, fear and duration 47 of labour (Fairbrother et al., 2008; Hall et al., 2009), and prematurity (Ko, Wu, \& Chang, 2002). 48 During the postpartum period, fatigue has been linked to depression (Wade, Giallo \& Cooklin, 49 2012), sleep alterations (Hung \& Chen, 2014), breastfeeding difficulties (Wambach, 1998), and 50 problems bonding with the baby (Lai et al., 2014). In early parenting, it has been found that higher 51 parental fatigue is associated with lower parental sense of confidence, higher parenting stress and

2 less optimal parenting behaviours (Cooklin, Giallo, \& Rose, 2012).

53 Fatigue is a symptom linked to the process of continual adaptation that begins during pregnancy 54 and continues through the transition to motherhood (Runquist, 2007). Various instruments have 55 been used previously during pregnancy and postpartum to measure fatigue, depending on whether 56 the aim was to obtain broad and detailed information about the experience of fatigue, using 57 multidimensional scales, or to measure the intensity or severity of fatigue, using unidimensional 58 scales (Dittner, Wessely, \& Brown, 2004; Whitehead, 2009). The Fatigue Assessment Scale (FAS) 59 (Michielsen, De Vries, \& Van Heck, 2003) is a tool that provides information about the physical 60 and psychological aspects of fatigue, and provides a single overall score measuring its intensity. 61 The FAS offers adequate characteristics as an instrument that measures patient reported outcomes 62 (U.S. Department of Health and Human Services FDA Center for Drug Evaluation and Research, 
63 2006), and this is because it is brief, it does not overburden the respondent, it has the capacity to 64 discriminate between cases and non cases in clinical groups, it is sensitive to change when 65 evaluating interventions, and it has good psychometric properties.

66 The FAS is a self-reported questionnaire consisting of 10 items with a 5-point Likert response 67 scale ranging from " $1=$ never to " $5=$ Always". Five items reflect the physical component and five 68 the psychological component.

69 Of these items, eight reflects negative aspects on fatigue states $(1,2,3,5,6,7,8,9)$. The remaining 70 two items (\#4 and \#10) are worded inversely and are given reverted scores, indicating energy 71 states. The FAS has been used in the context of postpartum and early parenthood among mothers 72 (Giallo, Wade, \& Kienhuis, 2014) and fathers (Mellor \& St. John, 2012; Seymour et al., 2014).

73 The Cronbach's alpha coefficient of the scale is above 0.80. The factor structure of the FAS has 74 been considered unidimensional (Michielsen et al., 2004). However, some studies have described 75 problems with two of the items, \#4 and \#10 (Kalkanis, Yucel, \& Judson, 2013; Giallo et al., 2014), 76 which negatively affect the internal consistency of the scale. This is probably caused by the 77 negative wording (Giallo et al., 2014). Furthermore, the suitability of the content of item \#10, 78 pertaining to problems of concentrating, is under debate, owing to the possibility that this item is 79 measuring depression (De Vries, Van der Steeg, \& Roukema, 2010).

80 Given the importance of fatigue as a symptom during pregnancy and postpartum, various 81 international (World Health Organization, 2013) and national (Ministerio de Sanidad, Servicios 82 Sociales e Igualdad, 2014) guidelines recommend it should be studied in motherhood in general, 83 and particularly during the postpartum period. However, fatigue has been studied very little in the 84 Spanish population, especially in the context of motherhood. There are no publications regarding 85 a Spanish version of the FAS. Hence, this study aims to: (1) validate the FAS at a linguistic level, 86 (2) test its factorial structure, and (3) provide evidence of convergent validity, discriminant 87 validity, as well as discriminative validity (comparisons between known groups). 


\section{Materials \& Methods.}

$90 \quad 2.1$ Design

91 An instrumental design (Dios \& Melendez, 2005) was conducted, including a two-phase process.

92 First, the scale was translated into Spanish and the content validity was examined, and second, 93 testing was conducted to establish reliability and validity.

94 2.2 Participants

95 96

A convenience sample of 870 women was recruited from 17 regional hospitals in Eastern Spain between January 2014 and June 2014 during their in-hospital postpartum stay. All participants were able to read and write in Spanish. Multiple pregnancies along with severe health problems in the new-born, and processes that required the new-born to be taken into a neonatal unit during the time the mother was hospitalized were excluded.

\subsection{Translation and cultural validity}

Permission from the authors of the FAS was obtained in order to use and translate it into Spanish. The translation was developed by forward-backward translations from the original English version (Muñiz, Elosua, \& Hambleton, 2013). There were two independent translations, developed by two native Spanish-speaking translators fluent in English. Translators were asked to grade the difficulty of the translation using a numeric rating scale from 1 (not at all difficulty) to 10 (maximum difficulty) and describe the changes they made to each item during translation in order to maintain the semantic equivalence. Both versions were compared by a panel of four experts consisting of two midwives and two psychologists and, after having reached a consensus, the instrument was prepared in a single document. This consensus version was then translated back to English, by two native English-speaking translators fluent in Spanish, without prior knowledge of the original version. This was then compared with the original, to ensure conceptual and semantic equivalence. The expert panel agreed that the Spanish version demonstrated semantic and grammatical equivalence, and one of the original English authors confirmed this aspect. Cognitive interviews of the Spanish version of the FAS was performed with ten pregnant and ten puerperal women to evaluate the adequacy of the format and presentation as well as to assess the clarity and interpretation of each item and response option.

\subsection{Data collection}


118 Participants completed a battery of self-administered questionnaires on the day of their hospital

119 discharge. The battery included, in addition to the FAS-e, a questionnaire on sociodemographic

120 variables and items generated ad hoc, using an analogical visual 100-point scale to obtain

121 information about: 1) perceived pain related to their labour or caesarean in the last 24 hours and,

122 2) difficulty breastfeeding during their stay in hospital. The level of maternal self-efficacy to

123 breastfeed was also measured by means of the short form of the Breast Feeding Self Efficacy Scale

124 (BSES-SF) (Oliver-Roig et al., 2012). Data on obstetric variables at discharge were also collected

125 from clinical records.

126 2.4 Data analysis

127 We calculated the mean and standard deviation of the item scores and, in order to assess floor and 128 ceiling effects, the proportion of respondents with the lowest or highest possible score were 129 described. We calculated missing data from each item to assess acceptability.

130 To examine the factorial structure of the scale we included participants who responded to all items 131 of the FAS scale $(\mathrm{n}=803)$. Also, as a cross-validation strategy, we randomly divided the sample 132 into two sub-samples of approximately equal size, one for development and another for validation. 133 We used exploratory factor analysis (EFA) and confirmatory factor analysis (CFA) with the first 134 sample. The CFA models examined were based on previous findings on the FAS scale and on the 135 EFA and CFA results themselves (examination of the modification indexes, the error terms 136 correlations, and factor loadings). According to the FAS scale background, we examined models 137 of a single factor (the model with the highest theoretical and empirical support), two correlated 138 factors (physical fatigue and mental fatigue), and variations of these models allowing correlated 139 errors (two items of the scale had a positive wording, i.e. positive states of fatigue, and the rest, 140 eight items, a negative wording, i.e., negative states of fatigue). In addition to these more 141 traditional models, we used bifactorial models. These models stipulate a general factor and one 142 or several specific factors, constrained as not correlated with each other, allowing to identify if the 143 general factor explains the greater part of the variance in spite of the specific factors (Deng, Guyer, $144 \&$ Ware, 2015). These models have been specifically recommended to examine whether a measure 145 is sufficiently one-dimensional in the presence of secondary dimensions (i.e., can it be assessed as 146 an essentially unidimensional measure or not, if secondary dimensions such as physical and mental 147 subdomains of a general measure of fatigue, or if the effects of wording of items distort the 
148 interpretation of the scale). With the second sample, we examined the robustness of the CFA

149 models analysed with the first sample. Due to the ordinal nature of the items and the non-normality

150 of their distributions we used methods suitable for categorical data, i.e., polychoric correlation

151 matrices, DWLS estimator in CFA and minimum residual method (MinRes) in EFA.

152 As indexes of goodness of fit of the models we use: the comparative fit index (CFI); goodness of 153 fit index (GFI), the root mean square error of approximation (RMSEA), and the standardized root 154 mean square residual (SRMR). Guidelines suggest that models with CFI and GFI close to 0.95 or 155 higher, RMSEA close to 0.06 or lower, and SRMR close to 0.08 or lower represent good-fitting 156 models. (Hu \& Bentler, 1999).

157 Reliability of the translated FAS-e was assessed using the following criteria: Cronbach's alpha 158 coefficient, adjusted item-total correlation, and estimation of $\alpha$ when an item was removed from 159 the scale.

160 Further, evidence of discriminant validity and relationships with other variables according to 161 previous evidence was obtained. The total scores from the FAS Scale were expected to be higher 162 for mothers having undergone a caesarean section (Cheng et al., 2014; Kilic et al., 2015; Lai et al., 163 2014; Rowlands \& Redshaw, 2012) and experienced increased pain in the postpartum period 164 (Fishbain et al., 2003; Wambach, 1998). It was also expected that women who experienced 165 breastfeeding problems or who had lower levels of self-efficacy in breastfeeding would obtain 166 higher scores on the FAS scale, given that breastfeeding is one of the main care demands of the 167 postpartum period (Dennis, 1999; Wambach, 1998).

168 Scores on the FAS scale were also evaluated for other sociodemographic types of variables, such 169 as age, or obstetric variables object of controversy in previous studies with regard to their 170 relationship with fatigue. The Kolmogorov-Smirnov test was used to compare the FAS-e score 171 distribution with the normal distribution of variables. The Student's T test and Pearson correlations 172 were used to compare hypotheses. The LISREL software (Jöreskog, \& Sörbom, 2006) was used 173 for all these factorial analyses. Other analyses were conducted using SPSS 22.0 (IBM Corp., 2013).

\section{$174 \quad 2.5$ Ethical aspects:}

175 The women in the sample were approached by a research assistant and informed about the study.

176 When the women expressed an interest in participating in the study, a screening interview was 
177 performed and, if eligible, written consent to participate was obtained. Approval was received from

178 the research ethics committee of the Directorate General of Public Health and Public Health 179 Research Center ("Dirección General de Salud Pública y Centro Superior de Investigación en 180 Salud Pública") of the Valencian Community (Spain).

\section{3. Results.}

182 3.1 Semantic equivalence

183 None of the 10 items on the scale were considered inappropriate for the Spanish context. It was 184 not necessary to make any changes to any of the 10 items. According to the translators' evaluation, 185 the average difficulty of the translation by item was 1.8 ( $\mathrm{SD}=0.4$, range 1.25 to 2.50 ), and did not 186 rise above 4 for any item on the established scale of 1-10.

187 Item \#1 "I am bothered by fatigue" sparked debate among the translators owing to the existence 188 of two possible terms for the translation of bothered, "molesta" or "preocupada". The 189 "preocupada" option was chosen, since "molesta" was considered to be more closely linked to 190 discomfort or pain rather than fatigue.

191 Reading and comprehension problems were not encountered in cognitive interviews, and the 192 average time for completion was 85.4 seconds ( $S D=25.3$, range $50-125)$.

193 3.2. Description of sample.

194 The average age of the sample was 32.4 years old ( $S D=4.9$, range 15-46). Table 1 provides the 195 other characteristics of the sample as well as percentiles of the FAS-e scores.

196 The questionnaire response rate was good. The percentage of data lost did not exceed 5\% for any 197 of the items on the scale. The mean total score obtained on the FAS-e score was 19.17 with a 198 standard deviation of 5.62 and the proportion of responses with the lowest possible score, "1", was 199 above $15 \%$ for all items (Table 2).

200 3.3. Factor analysis results, first subsample $(\mathrm{n}=406)$

201 The EFA analysis on the first sub-sample $(\mathrm{n}=406)$ identified three possible factor solutions: 202 unifactorial, two factors and three factors (the first six components had the following eigenvalues: $2034.72,1.17,0.83,0.78,0.61,0.54)$. In the unifactorial solution, the loadings of the items ranged 204 between 0.34 and 0.81 . The correlated two-factor solution (Promax rotation, $\mathrm{r}=0.46$ ) brought 
205 together the eight items with negative wording in one factor and the two items with positive 206 wording in the other, with a clear simple structure. The three-factor solution brought together four 207 items of physical fatigue in one factor, four items of mental fatigue in a second factor and the two 208 items with positive wording in the third factor. The four-factor solution was uninterpretable and 209 clearly meant an overfactorization.

210 Table 3 describes the CFA models examined with the two sub-samples, as well as the results of 211 the fit indexes of the models. In general, the models examined were those stipulated a priori, since 212 the results of EFA and CFA did not provide any notable novelty (i.e., there was no indication to 213 estimate more error terms correlations between items than that stipulated a priori between error 214 terms of the two items with positive wording).

215 Regarding the CFA results, the one-dimension model (Model 1a) fitted well to the data, with 216 loadings ranging from 0.35 to 0.83 ; its fit improved slightly (Model 1b) when the correlation 217 between the error terms of items with positive wording $\left(\theta_{4,10}=0.34\right)$ was allowed. The correlated 218 two-factor model presented only slightly better indices than the unidimensional model and 219 estimated a very high correlation between both factors $(\mathrm{r}=0.88)$. As with the unidimensional 220 model, allowing the correlation between the errors of the two items with positive wording (Model 221 2b) enabled slightly improving the fit.

222 To what extent did the multidimensionality identified in these models, i.e., the effects of wording 223 and subdomains of mental and physical fatigue obscure a unidimensional interpretation of the FAS 224 scale? First, according to the results of the bifactorial models that included wording effects (models $2253 \mathrm{a}$ and $3 \mathrm{~b}$ ), although there was a secondary factor of positive wording (Model 3a), its existence 226 hardly had an influence on the loadings of these items in the general factor (their loadings were 227 approximately the same as those of the pure unidimensional model, Model 1a). Also, the model 228 including a specific factor composed of the eight items with negative wording (Model 3b) showed 229 the irrelevance of this specific factor (loadings not significant or less than 0.2 , except for one item, 230 in the negative wording specific factor and loadings in the general factor very close to those of the 231 unidimensional model). Therefore, the wording of the items did not interfere with the 232 unidimensional interpretation of the scale. Secondly, regarding the influence of mental and 233 physical fatigue subdomains, Model 4a did not converge but Model 4b, including the specific 234 factors of mental fatigue and physical fatigue in addition to the estimation of the correlated error 
235 terms between items \#4 and \#10, did converge. This model achieved the best fit indexes (it 236 incorporated all sources of variation found: general fatigue factor, positive wording of two items 237 and fatigue subdomains) and showed a strong general factor (loadings were slightly lower than 238 those of the unidimensional model with a difference equal to or less than 0.05 in 9 items and the 239 largest difference was 0.08 in item 8), two weak specific factors (only one item in each specific 240 factor had loadings greater than 0.3 and an error terms correlation between items \#4 and \#10 of 241 0.33). In summary, the FAS scale was sufficiently unidimensional, despite the presence of method 242 effects (positive wording) and effects related to the subdomains of fatigue.

243 3.4. Factor analysis results, second subsample $(\mathrm{n}=397)$.

244 The results on the second (validation) sub-sample $(\mathrm{n}=397)$ corroborated those of sub-sample 1, 245 however in this case the effects of method were somewhat smaller and those of subdomains 246 somewhat larger (i.e., the correlation between physical and mental fatigue was 0.81 , compared to $247 \quad 0.87$ in the first sub-sample).

\section{3.5. Internal consistency reliability}

249 Cronbach's alpha was .80. FAS-e items reliability results, means and standard deviations are 250 provided in Table 2. The alpha value decreases if any item is eliminated, with the exception of 251 eliminating item $\# 10$, which increases it to .82 . The item-total correlations for the 10 items were 252 all $>.30$, positive and significant in this study, and ranged between .32 and .61 .

253 3.6. Discriminant validity, comparisons between known groups and correlations with other 254 variables.

255 Total score average of the FAS-e was $19.17(S D=5.60)$, showing a non normal distribution $(z=2.46$, $256 p=.01)$. The average FAS-e score was $18.92(S D=5.4)$ for women with vaginal delivery and 20.17 257 ( $S D=6.15)$ for women with caesarean, reflecting significant differences between the FAS-e scores $258(t=-2.53 ; p<0.05)$. The Pearson correlation coefficient between pain intensity during postpartum 259 (Likert 0-100) and total FAS-e score was .18; $p<.01$. The Pearson correlation coefficient between 260 breastfeeding difficulties (Likert 0-100) and FAS-e score was .12; $p<.01$. The Pearson correlation 261 coefficient between BSES-SF and FAS-e score was $-.25 ; p<.01$. No differences were found related 262 to age $(r=-0.04 ; p=.24)$, parity $(t=87 ; p=.38)$ and type of feeding at discharge $(t=-1.75 ; p=.08)$.

\section{4. Discussion.}


264 In this study, we developed the Spanish version of the FAS, provided evidence of validity and 265 reliability, and analysed its factorial structure, considering physical and mental subdomains and 266 the effect of the wording of items, in a sample of postpartum women at hospital discharge.

267 The translation process was systematic and rigorously conducted to ensure that equivalence was 268 established. Only one item (\#1) sparked debate in translation owing to the dual possible translation 269 of "bothered" as either "molesta" or "preocupada". Once consensus was reached regarding its 270 translation, it did not present any problems in the pilot test or the definitive sample.

271 The scale presented a floor effect for all items. This greater proportion of responses at lower levels 272 of fatigue in the sample of this study could be due to the fact that the participants were young 273 healthy women. Other possible causes for the lower level of fatigue among the sample studied are 274 that the data were obtained in the morning, when levels of fatigue are lower (Elek, Hudson, \& 275 Fleck, 2002), and on the date of discharge, so participants might have had a tendency to report less 276 fatigue, influenced by their imminent return home with their baby (Fredrickson,2000; Tzeng et al., 277 2008). No evidence was provided on the floor effect in the scoring of items in previous studies that 278 used the FAS scale on a sample of postpartum women.

279 The findings on the dimensionality of the FAS scale indicate that it was sufficiently 280 unidimensional, despite the presence of a certain multidimensionality derived from the positive 281 wording effects of two of the items, in contrast to the negative wording of the rest of the eight 282 items, and despite the effects of the subdomains of the scale, i.e. physical fatigue and mental 283 fatigue. We modelled these effects in two ways: (i) through factor models with correlated errors 284 (to estimate the effects of item wording) as well as correlated two-factor models (to estimate the 285 effects of fatigue subdomains), and (ii) bifactor models that incorporated both types of effects as 286 specific factors, in addition to a general factor. The findings of the bifactorial models revealed that 287 these sources of variation were secondary and in no way distorted the interpretation of the scale as 288 a solid one-dimensional measure. Unidimensionality of the FAS scale is a general finding in the 289 literature (Michielsen et al, 2003; Michielsen et al., 2004) as well as a specific finding when studied 290 in a population similar to ours, i.e., postpartum women (Fairbrother et al., 2008; Tsai et al., 2014).

291 In addition, our findings are also compatible with the content specifications of the scale, since the 292 two subdomains, mental and physical fatigue, underlie but do not distort the unidimensionality of 293 the scale. And finally, our findings corroborate the effects that introduce the contrast of the positive 
294 wording of two items versus the negative wording of the rest of the items on the scale (Kalkanis 295 et al., 2013). Although we performed a methodological interpretation of the wording effects of the 296 items, a more substantive interpretation is also plausible (Deng, Guyer, \& Ware, 2015): items with 297 negative wording would be indicators of fatigue, whereas items with positive wording would be 298 indicators of energy (and both would be indicators of the same construct: vitality).

299 The FAS-e offers acceptable internal consistency. Cronbach's alpha for the FAS-e (.81) was lower 300 than the original version (.90), but higher than the minimum acceptable level of .70, and similar to 301 the value obtained in other populations/samples (De Vries et al., 2004; Michielsen et al., 2003).

302 In this study, as in others (Lai et al., 2014; Cheng et al., 2014; Kilic et al.,2015; Rowlands \& 303 Redshaw, 2012), women who had undergone a caesarean showed higher fatigue than those who 304 had vaginal deliveries. Additionally, as described previously, women with a higher level of pain 305 during the postpartum period (Fishbain et al., 2003; Wambach, 1998) or higher perceived difficulty 306 with breastfeeding (Wambach, 1998) were more fatigued. Furthermore, the data confirmed, as 307 previously suggested (Denis,1999), that fatigue was one of the symptoms relating to self-efficacy 308 in breastfeeding.

309 Increased age has been associated with greater obstetric problems and, therefore, high levels of 310 fatigue postpartum (Taylor \& Johnson, 2013). First-time mothers can present greater fatigue 311 related with longer labours and inexperience in parenting (Tzeng et al., 2008; Taylor \& Johnson, 312 2013). Our data did not suggest differences in levels of fatigue relating to these variables. The fact 313 that the data were obtained prior to postpartum discharge and before the women had had to cope 314 with the challenges of daily life and the re-establishment of family roles, could explain the lack of 315 differences relating to the parity variable. As for the influence of the mother's age, another recent 316 study similarly found no significant differences in levels of postpartum fatigue in primaparous 317 women according to age (Tsuchiya et al., 2015).

318 In the future, it would be desirable to test the performance of the instrument at different points 319 during the postpartum period, or following interventions, to ascertain the instrument's sensitivity 320 to change in this population group. It would also be interesting to explore how the instrument 321 performs for fathers, who are also affected by childbirth.

\section{Conclusions.}


323 The Spanish version of the FAS (FAS-e) has been obtained and its preliminary validation has been 324 established. The FAS-e is a sufficiently unidimensional, valid, and reliable tool to study fatigue in 325 postpartum women in Spain.

326 


\section{References}

328 Aaronson, L. S., Teel, C. S., Cassmeyer, V., Neuberger, G. B., Pallikkathayil, L., Pierce, J., Press A.N., Williams P.D., Wingate, A. (1999). Defining and measuring fatigue. Journal of Nursing Scholarship, 31(1), 45-50. http://doi.org/10.1111/j.1547-5069.1999.tb00420.x

Cheng, C.-Y., \& Li, Q. (2008). Integrative Review of Research on General Health Status and Prevalence of Common Physical Health Conditions of Women After Childbirth. Women's Health Issues, 18(4), 267-280 http://doi.org/10.1016/j.whi.2008.02.004

335

336

337

338

339

340

341

342

343

344 345

346

347 348 349

350

351

352

353 354

Cheng, C.-Y., Chou, Y.-H., Wang, P., Tsai, J.-M. and Liou, S.R. (2014), Survey of trend and factors in perinatal maternal fatigue. Nursing \& Health Sciences, 17(1), 64-70. doi:10.1111/nhs. 12149

Cooklin, A. R., Giallo, R., \& Rose, N. (2012). Parental fatigue and parenting practices during early childhood: An Australian community survey. Child: Care, Health and Development, 38(5), 654-664. http://doi.org/10.1111/j.1365-2214.2011.01333.x

De Vries, J. , Van der Steeg, A. F., \& Roukema, J. A. (2010). Psychometric properties of the Fatigue Assessment Scale (FAS) in women with breast problems. International Journal of Clinical and Health Psychology, 10 (1), 125-139. Available in: http://www.redalyc.org/articulo.oa?id=33712017008

De Vries, J., Michielsen, H., Van Heck, G. L., \& Drent, M. (2004). Measuring fatigue in sarcoidosis: the Fatigue Assessment Scale (FAS). British Journal of Health Psychology, 9(Pt 3), 279-91. http://doi.org/10.1348/1359107041557048

Deng, N., Guyer, R., \& Ware, J. (2015). Energy, fatigue, or both? A bifactor modeling approach to the conceptualization and measurement of vitality. Quality of Life Research, 24(1), 8193. 10.1007/s11136-014-0839-9

Dennis, C.-L. (1999). Theoretical Underpinnings of Breastfeeding Confidence: A Self-Efficacy Framework. Journal of Human Lactation, 15(3), 195-201. http://doi.org/10.1177/089033449901500303

Dios, H.C. \& Melendez, C.P.(2005). Normas para el desarrollo y revisión de estudios instrumentales. International Journal of Clinical and Health Psychology, 5(3), 521-551. 
355 Dittner, A. J., Wessely, S. C., \& Brown, R. G. (2004). The assessment of fatigue: A practical

356

357

358

359

360

361

362

363

364

365

366

367

368

369

370

371

372

373

374

375

376

377

378

379

380

381

382

383 guide for clinicians and researchers. Journal of Psychosomatic Research, 56(2), 157-170. http://doi.org/10.1016/S0022-3999(03)00371-4

Elek, S. M., Hudson, D. B., \& Fleck, M. O. (2002). Couples' Experiences with Fatigue during the Transition to Parenthood. Journal of Family Nursing, 8(3), 221-240. http://doi.org/10.1177/107484070200800305

Fairbrother, N., Hutton, E. K., Stoll, K., Hall, W., \& Kluka, S. (2008). Psychometric evaluation of the Multidimensional Assessment of Fatigue scale for use with pregnant and postpartum women. Psychological Assessment, 20(2), 150-8. http://doi.org/10.1037/1040$\underline{3590.20 .2 .150}$

Fishbain, D. A., Cole, B., Cutler, R. B., Lewis, J., Rosomoff, H. L., \& Rosomoff, R. S. (2003). Is pain fatiguing? A structured evidence-based review. Pain Medicine, 4(1), 51-62. http://doi.org/10.1046/j.1526-4637.2003.03008.x

Fredrickson, B. L. (2000). Extracting meaning from past affective experiences: The importance of peaks, ends, and specific emotions. Cognition \& Emotion, 14(4), 577-606. http://doi.org/10.1080/026999300402808

Giallo, R., Wade, C., \& Kienhuis, M. (2014). Fatigue in mothers of infants and young children: factor structure of the fatigue assessment scale. Fatigue: Biomedicine, Health \& Behavior, 2(3), 119-131. http://doi.org/10.1080/21641846.2014.925326

Hall, W. , Hauck, Y. L., Carty, E. M., Hutton, E. K., Fenwick, J., \& Stoll, K. (2009). Childbirth fear, anxiety, fatigue, and sleep deprivation in pregnant women. Journal of Obstetric, Gynecologic, and Neonatal Nursing, 38(5), 567-76. http://doi.org/10.1111/j.1552$\underline{6909.2009 .01054 . \mathrm{x}}$

Hu, L., \& Bentler, P. M. (1999). Cutoff criteria for fit indexes in covariance structure analysis: Conventional criteria versus new alternatives. Structural Equation Modeling: A Multidisciplinary Journal, 6(1), 1-55 http://doi.org/10.1080/10705519909540118

Hung, H.-M., \& Chen, C.-H. (2014). Sleep quality in postpartum women: exploring correlation with childbirth experience and household work. The Journal of Nursing Research, 22(1), 20-7. http://doi.org/10.1097/jnr.0000000000000015 
384 IBM Corp. Released 2013. IBM SPSS Statistics for Windows, Version 22.0. Armonk, NY: IBM $385 \quad$ Corp.

386 Jöreskog, K. G., \& Sörbom, D. (2006). LISREL 8.80 [Computer software]. Chicago: Scientific 387 Software International.

388 Kalkanis, A., Yucel, R. M., \& Judson, M. A. (2013). The internal consistency of PRO fatigue 389 instruments in sarcoidosis: superiority of the PFI over the FAS. Sarcoidosis, Vasculitis, 390 and Diffuse Lung Diseases, 30(1), 60-4.

391 Kilic, M., Ozorhan E.Y., Apay S.E., Capik A., Agapinar, S., \& Ozkan, H.(2015). Comparison of 392 Fatigue Levels of Postratum Women According to the birth method. International Journal 393 of Caring Sciencies, 8(1), 124-131.

394 Ko, Y.-L., Wu, Y.-C., \& Chang, P.-C. (2002). Physical and social predictors for pre-term births 395 396 and low birth weight infants in Taiwan. Journal of Nursing Research, 10(2), 83-89. doi:10.1097/01.JNR.0000347586.08328.d6

397

Lai, Y., Hung, C., Stocker, J., Chan, T., \& Liu, Y. (2014). Postpartum fatigue , baby-care 398 activities , and maternal - infant attachment of vaginal and cesarean births following

399 rooming-in. Applied Nursing Research. 28(2),116-120.

400 http://doi.org/10.1016/j.apnr.2014.08.002

401

402

403 404 405

406 407 408 409 410

McQueen, A., \& Mander, R. (2003). Tiredness and fatigue in the postnatal period. Journal of Advanced Nursing, 42(5), 463-9. doi: 10.1046/j.1365-2648.2003.02645.x

Mellor, G., \& John, W. S. (2012). Fatigue and work safety behavior in men during early fatherhood. American journal of men's health, 6(1), 80-88. doi: $10.1177 / 1557988311423723$

Michielsen, H. J., De Vries, J., \& Van Heck, G. L. (2003). Psychometric qualities of a brief selfrated fatigue measure: The Fatigue Assessment Scale. Journal of Psychosomatic Research, 54(4), 345-352. http://doi.org/10.1016/S0022-3999(02)00392-6

Michielsen, H. J., De Vries, J., Van Heck, G. L., Van de Vijver, F. J. R., \& Sijtsma, K. (2004). Examination of the Dimensionality of Fatigue: The Construction of the Fatigue 
411

412

413

414

415

416

417

418

419

420

421

422

423

424

425

426

427

428

429

430

431

432

433

434

435

436

437

438

439

Assessment Scale (FAS). European Journal of Psychological Assessment, 20(1) 39-48. http://doi.org/10.1027/1015-5759.20.1.39

Ministerio de Sanidad, Servicios Sociales e Igualdad. (2014). Guía de práctica clínica de atención en el embarazo y puerperio. Madrid. Retrieved from http://www.msssi.gob.es/organizacion/sns/planCalidadSNS/pdf/Guia_practica_AEP.pdf on May 24,2016.

Muñiz, J., Elosua, P., \& Hambleton, R. K. (2013). Directrices para la traducción y adaptación de los tests: segunda edición. Psicothema, 25(2), 151-157. http://doi.org/10.7334/psicothema2013.24

National Library of Medicine. (2016). Medical Subject Headings. Retrieved from http://www.ncbi.nlm.nih.gov/mesh/68005221 on May 7, 2016.

Oliver-Roig, A., D’Anglade-González, M. L., García-García, B., Silva-Tubio, J. R., RichartMartínez, M., \& Dennis, C. L. (2012). The Spanish version of the Breastfeeding SelfEfficacy Scale-Short Form: Reliability and validity assessment. International Journal of Nursing Studies, 49(2), 169-173. http://doi.org/10.1016/j.ijnurstu.2011.08.005

Rowlands, I. J., \& Redshaw, M. (2012). Mode of birth and women's psychological and physical wellbeing in the postnatal period. BMC Pregnancy Childbirth, 12(1), 138. http://doi.org/10.1186/1471-2393-12:138

Runquist, J. (2007). Persevering through postpartum fatigue. JOGNN - Journal of Obstetric, Gynecologic, and Neonatal Nursing, 36(1), 28-37. http://doi.org/10.1111/j.1552$\underline{6909.2006 .00116 . x}$

Seymour, M., Dunning, M., Cooklin, A., \& Giallo, R. (2014). Socioecological factors associated with fathers' well-being difficulties in the early parenting period. Clinical Psychologist, 18(2), 63-73. http://doi.org/10.1111/cp.12016

Taylor, J., \& Johnson, M. (2013). The role of anxiety and other factors in predicting postnatal fatigue: From birth to 6 months. Midwifery, 29(5), 526-534. http://doi.org/10.1016/j.midw.2012.04.011

Tiesinga, L. J., Dassen, T. W., \& Halfens, R. J. (1998). DUFS and DEFS: development, reliability and validity of the Dutch Fatigue Scale and the Dutch Exertion Fatigue Scale. 
440

441

442

443

444

445

446

447

448

449

450

451

452

453

454

455

456

457

458

459

460

461

462

463

464

465

466

467

468

International Journal of Nursing Studies, 35(1-2), 115-123. http://doi.org/10.1016/S00207489(98)00005-4

Tsai, S.-Y., Shun, S.-C., Lai, Y.-H., Lee, Y.-L., \& Lee, S.-Y. (2014). Psychometric evaluation of a Chinese version of the Lee Fatigue Scale-Short Form in women during pregnancy and postpartum. International Journal of Nursing Studies, 51(7), 1027-35.

http://doi.org/10.1016/j.ijnurstu.2013.10.023

Tsuchiya, M., Mori, E., Sakajo, A., Maekawa, T., Iwata, H., Maehara, K., Morita, A., Ozawa, H., Mochizuki, Y., Aoki, K., Makaya, M., \& Tamakoshi, K. (2015).Age-specific determinants of post-partum fatigue in primiparous women. Japan Journal of Nursing Science, 13: 8394. doi: $10.1111 /$ jjns. 12089 .

Tzeng, Y.-L., Chao, Y.-M. Y., Kuo, S.-Y., \& Teng, Y. K. (2008). Childbirth-related fatigue trajectories during labour. Journal of Advanced Nursing, 63(3), 240-9. http://doi.org/10.1111/j.1365-2648.2008.04732.x

U.S. Department of Health and Human Services FDA Center for Drug Evaluation and Research, U.S. Department of Health and Human Services FDA Center for Biologics Evaluation and Research, \& U.S. Department of Health and Human Services FDA Center for Devices and Radiological Health. (2006). Guidance for industry: patient-reported outcome measures: use in medical product development to support labeling claims: draft guidance. Health and Quality of Life Outcomes, 4, 79. http://doi.org/10.1186/1477-7525-4-79

Wade C, Giallo R., \& Cooklin A. (2012). Maternal Fatigue and depression: Identifying vulnerability and relationship to early parenting practices. Advances in mental health, 10(3): $277-291$

Wambach, K. A. (1998). Maternal fatigue in breastfeeding primiparae during the first nine weeks postpartum. Journal of human Lactation,14(3), 219-29

Doi:10.1177/089033449801400311

Whitehead, L. (2009). The measurement of fatigue in chronic illness: a systematic review of unidimensional and multidimensional fatigue measures. Journal of Pain and Symptom Management, 37(1), 107-28. http://doi.org/10.1016/j.jpainsymman.2007.08.019

World Health Organization. (2013). WHO recomenatations on postnatal care of the mother and 
newborn. Retrieved from

470

http:/www.who.int/maternal child adolescent/documents/postnatal-care-

471 recommendations/en/ on August 15,2016 
Table $\mathbf{1}$ (on next page)

Characteristics of the study sample. 
1 Characteristics of the study sample $(n=870)$.

2

\begin{tabular}{|c|c|}
\hline Variable & $\mathrm{n}(\%)$ \\
\hline Country & \\
\hline Spain & $751(86.5)$ \\
\hline Other countries & $117(13.5)$ \\
\hline \multicolumn{2}{|l|}{ Education Level } \\
\hline Primary school & $378(44.3)$ \\
\hline High school/University degree & $476(55.7)$ \\
\hline \multicolumn{2}{|l|}{ Marital status } \\
\hline De facto or married & $702(82.6)$ \\
\hline Single & $148(17.4)$ \\
\hline \multicolumn{2}{|l|}{ Living with partner } \\
\hline Yes & $817(95.9)$ \\
\hline No & $35(4.1)$ \\
\hline \multicolumn{2}{|l|}{ Income } \\
\hline$<12000 \in$ & $280(35.6)$ \\
\hline$>12000 \in$ & $506(64.4)$ \\
\hline \multicolumn{2}{|l|}{ Parity } \\
\hline Primiparae & $428(49.2)$ \\
\hline Multiparae & $442(50.8)$ \\
\hline \multicolumn{2}{|l|}{ Mode of delivery } \\
\hline Vaginal delivery & $692(79.6)$ \\
\hline Caesarean delivery & $177(20.4)$ \\
\hline \multicolumn{2}{|l|}{ Breastfeeding initiation } \\
\hline Yes & $770(88.7)$ \\
\hline No & $98(11.3)$ \\
\hline Percentiles of the FAS-e scores & Score \\
\hline 10 & 13 \\
\hline 20 & 14 \\
\hline 25 & 15 \\
\hline 30 & 16 \\
\hline 40 & 17 \\
\hline 50 & 19 \\
\hline 60 & 20 \\
\hline 70 & 21 \\
\hline 75 & 22 \\
\hline 80 & 23 \\
\hline 90 & 26 \\
\hline
\end{tabular}




\section{Table 2(on next page)}

FAS-e items reliability results, floor and ceiling effects, means (M) and standard deviation (SD). 


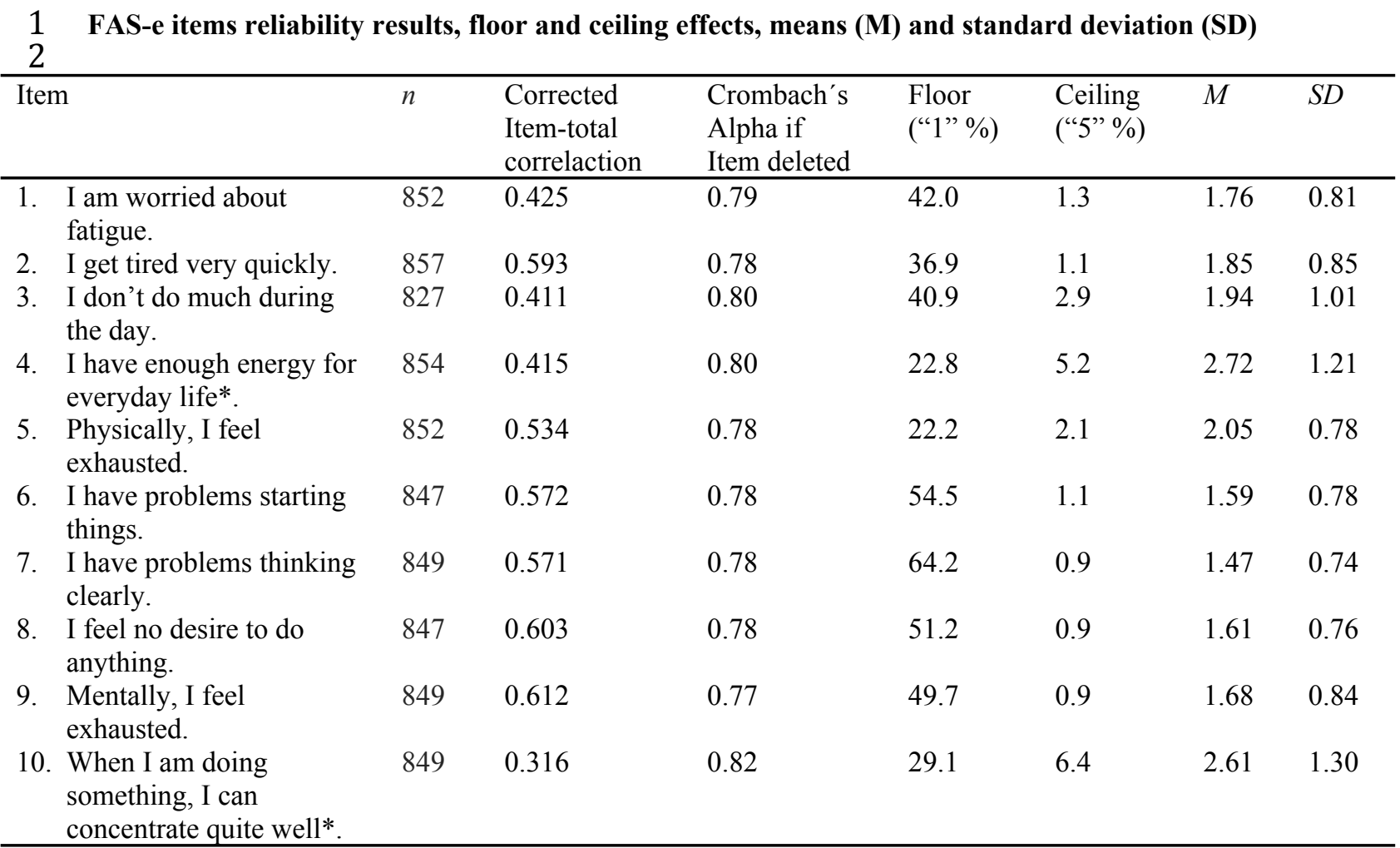

3 * Items \#4 and \#10 have been reverted before carrying all computations. 


\section{Table 3 (on next page)}

Confirmatory factor analyses of the Fatigue Assessment Scale: Overall model fit. 
1 Confirmatory factor analyses of the Fatigue Assessment Scale: Overall model fit.

2

\begin{tabular}{|c|c|c|c|c|c|c|}
\hline Model & $\chi^{2}$ s-B & $d f$ & CFI & GFI & SRMR & RMSEA \\
\hline \multicolumn{7}{|c|}{$\begin{array}{l}\text { Subsample 1, } \\
\mathrm{N}=406\end{array}$} \\
\hline Model 1a & 108.8 & 35 & 0.98 & 0.99 & 0.064 & 0.072 \\
\hline Model 1b & 83.8 & 34 & 0.98 & 0.99 & 0.048 & 0.060 \\
\hline Model 2a & 87.5 & 34 & 0.98 & 0.99 & 0.059 & 0.062 \\
\hline Model 2b & 61.44 & 33 & 0.99 & 0.99 & 0.040 & 0.046 \\
\hline Model 3a & 84.99 & 33 & 0.98 & 0.99 & 0.048 & 0.062 \\
\hline Model 3b & 50.94 & 25 & 0.99 & 1.00 & 0.035 & 0.051 \\
\hline Model 4a & - & - & - & - & - & - \\
\hline Modelo 4b & 38.5 & 24 & 1.00 & 1.00 & 0.032 & 0.039 \\
\hline \multicolumn{7}{|c|}{$\begin{array}{l}\text { Subsample 2, } \\
\mathrm{N}=397\end{array}$} \\
\hline Model 1a & 119.1 & 35 & 0.97 & 0.98 & 0.064 & 0.078 \\
\hline Model 1b & 114.1 & 34 & 0.97 & 0.99 & 0.064 & 0.077 \\
\hline Model 2a & 73.0 & 34 & 0.99 & 0.99 & 0.052 & 0.054 \\
\hline Model 2b & 62.4 & 33 & 0.99 & 0.99 & 0.045 & 0.047 \\
\hline Model 3a & 112.7 & 33 & 0.97 & 0.99 & 0.060 & 0.078 \\
\hline Model 3b & 50.94 & 25 & 0.99 & 1.00 & 0.035 & 0.051 \\
\hline Model 4a & - & - & - & - & - & - \\
\hline Model 4b & 41.63 & 24 & 0.99 & 0.99 & 0.033 & 0.043 \\
\hline
\end{tabular}

$\chi^{2}$ s-B Chisquare Satorra-Bentler

CFI -Comparative Fit Index

GFI- Goodness of Fit Index

RMSEA- Root Mean Square Error of Approximation

SRMR- Standarized Root Mean Square Residual

Model 1a: one-dimensional model.

Model 1b: model 1a with correlated errors between items with positive wording, items \#4 and \#10.

Model 2a: two correlated factors: physical fatigue (five items) and mental fatigue (five items).

Model 2b: model 2a with correlated errors between items \#4 and \#10.

Model 3a: bifactorial model of a general factor and a specific factor with items with positive wording.

Model 3b: bifactorial model of a general factor and two specific factors, one with items with positive wording and the other with items with negative wording.

Model 4a: bifactorial model of a general factor and two specific factors, one for the items of physical fatigue and the other for those of mental fatigue. It did not converge in any of the two subsamples. 
19 Model 4b: model 4a with correlated errors between items \#4 and \#10. 